\title{
Quality of life and its predictors in adults with tuberous sclerosis complex (TSC): a multicentre cohort study from Germany
}

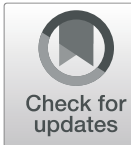

Johann Philipp Zöllner ${ }^{1,2}$, Nadine Conradi ${ }^{1,2}$, Matthias Sauter ${ }^{3}$, Markus Knuf ${ }^{4,5}$, Susanne Knake ${ }^{6}$, Gerhard Kurlemann ${ }^{7}$, Thomas Mayer ${ }^{8}$, Christoph Hertzberg ${ }^{9}$, Astrid Bertsche ${ }^{10}$, Ilka Immisch ${ }^{6}$, Karl Martin Klein ${ }^{1,2,11}$, Klaus Marquard ${ }^{12}$, Sascha Meyer ${ }^{13}$, Anna H. Noda ${ }^{1,2}$, Felix von Podewils ${ }^{14}$, Hannah Schäfer ${ }^{15,16}$, Charlotte Thiels ${ }^{17}$, Bianca Zukunft ${ }^{18}$, Susanne Schubert-Bast ${ }^{1,2,19}$, Janina Grau ${ }^{1,2}$, Laurent M. Willems ${ }^{1,2}$, Felix Rosenow ${ }^{1,2}$, Jens-Peter Reese ${ }^{20}$ and Adam Strzelczyk ${ }^{1,2,6^{*}}$ (D)

\begin{abstract}
Background: Tuberous sclerosis complex (TSC) is a monogenetic, multisystemic disease characterised by the formation of benign tumours that can affect almost all organs, caused by pathogenic variations in TSC1 or TSC2. In this multicentre study from Germany, we investigated the influence of sociodemographic, clinical, and therapeutic factors on quality of life (QoL) among individuals with TSC.
\end{abstract}

Methods: We assessed sociodemographic and clinical characteristics and QoL among adults with TSC throughout Germany using a validated, three-month, retrospective questionnaire. We examined predictors of health-related QoL (HRQoL) using multiple linear regression analysis and compared the QoL among patients with TSC with QoL among patients with other chronic neurological disorders.

Results: We enrolled 121 adults with TSC (mean age: $31.0 \pm 10.5$ years; range: 18-61 years, 45.5\% [ $n=55]$ women). Unemployment, a higher grade of disability, a higher number of organ manifestations, the presence of neuropsychiatric manifestations or active epilepsy, and a higher burden of therapy-related adverse events were associated with worse QoL, as measured by two QoL instruments (EuroQoL-5 dimensions [EQ-5D] and Quality of Life in Epilepsy Patients [QOLIE-31]). Neuropsychiatric and structural nervous system manifestations, the number of affected organs, and therapy-related adverse events were also associated with higher depression, as measured by the Neurological Disorders Depression Inventory for Epilepsy (NDDI-E). In multiple regression analysis, more severe therapyrelated adverse events (large effect, $p<0.001$ ), active epilepsy (large effect, $p<0.001$ ), and neuropsychiatric manifestations (medium effect, $p=0.003$ ) were independently associated with worse HRQoL, explaining $65 \%$ of the variance $(p<0.001)$. The HRQoL among patients with active TSC-associated epilepsy was worse than that among patients with drug-refractory mesial temporal lobe epilepsy $(p<0.001)$, and the generic QoL among patients with more than three TSC organ manifestations was similar to those of patients with severe migraine and uncontrolled asthma.

\footnotetext{
* Correspondence: strzelczyk@med.uni-frankfurt.de

${ }^{1}$ Epilepsy Center Frankfurt Rhine-Main, Center of Neurology and

Neurosurgery, Goethe-University Frankfurt, Schleusenweg 2-16 (Haus 95),

60528 Frankfurt am Main, Germany

${ }^{2}$ Center for Personalized Translational Epilepsy Research (CePTER),

Goethe-University Frankfurt, Frankfurt am Main, Germany

Full list of author information is available at the end of the article
}

(c) The Author(s). 2021 Open Access This article is licensed under a Creative Commons Attribution 4.0 International License, which permits use, sharing, adaptation, distribution and reproduction in any medium or format, as long as you give appropriate credit to the original author(s) and the source, provide a link to the Creative Commons licence, and indicate if changes were made. The images or other third party material in this article are included in the article's Creative Commons licence, unless indicated otherwise in a credit line to the material. If material is not included in the article's Creative Commons licence and your intended use is not permitted by statutory regulation or exceeds the permitted use, you will need to obtain permission directly from the copyright holder. To view a copy of this licence, visit http://creativecommons.org/licenses/by/4.0/. 
Conclusions: Active epilepsy, neuropsychiatric manifestations (such as anxiety and depression), and therapy-related adverse events are important independent predictors of worse quality of life among adults with TSC. Generic quality of life in TSC with several manifestations is similar to uncontrolled severe chronic diseases and significantly negatively correlates with TSC severity.

Trial registration: DRKS, DRKS00016045. Registered 01 March 2019.

Keywords: TSC, Tuberous sclerosis complex, Seizure, Epilepsy, Organ manifestations, Quality of life, Rare disease, EQ-5D, QOLIE-31, mTOR inhibitor

\section{Background}

Tuberous sclerosis complex (TSC) is a rare, multisystemic, monogenetic disorder. The incidence of definite or possible TSC is estimated at 1:6760 to $1: 13,520$ in Germany [1], and TSC prevalence was likely underestimated until recently due to clinical variability [1-4]. TSC is primarily caused by pathogenic variations in the TSC1 and TSC2 genes, causing the disinhibition of mechanistic target of rapamycin (mTOR) complex and leading to the dysregulation of cell metabolism, proliferation, and growth and ultimately the formation of benign tumours in multiple organ systems. TSC clinical manifestations vary throughout life, and initially present often in the heart, skin, and brain. Subsequent manifestations can appear in almost every organ, especially the brain, skin, kidneys, eyes, heart, and lungs, resulting in considerable interindividual phenotypic variability among individuals with TSC. Clinical manifestations can range from mild to sufficiently severe to require continuous nursing assistance [2, 5]. Approximately 85$96 \%$ of individuals with TSC suffer from structural epilepsy due to the formation of cortical tubers or other malformations $[5,6]$. Seizures often manifest within the first 6 months after birth [7]. Other common initial findings include hypomelanotic macules on the skin and cardiac rhabdomyoma, which is often detected prenatally during routine ultrasound examinations and is strongly associated with a TSC diagnosis [5]. Neuropsychiatric problems, including intellectual disability, autism, sleep difficulties, aggression, and anxiety and depression in adults, frequently occur in TSC [5]. Renal angiomyolipoma (AML) and brain subependymal giant cell astrocytoma (SEGA) often present during adolescence, and AML tend to grow during adolescence and adulthood, necessitating life-long surveillance [8]. Pulmonary lymphangioleiomyomatosis almost exclusively affects adult women with TSC [9].

Due to the multifaceted manifestations of TSC, the burden of illness is considerable [10-13]. Studies examining the quality of life (QoL) among those with TSC remain rare. A recent UK study found that QoL was impaired in all individuals with TSC, regardless of the presence of epilepsy and learning disabilities, with the psychosocial domain being the most affected [14]. Another study found worse QoL among those with frequent and more severe seizures [15], supported by a recent Dutch study [16].

However, few studies to date have investigated which aspects of TSC have the strongest effects on QoL. Thus, the present study aimed to provide a comprehensive analysis of QoL among adults with TSC and expand the current knowledge regarding QoL and its predictors by surveying a large, multicentre sample of adults with TSC in Germany.

\section{Methods}

\section{Patients and survey methods}

The present study was designed as a cross-sectional, multicentre survey that enrolled individuals with TSC throughout Germany (Berlin, Bochum, Dresden [Radeberg], Frankfurt, Greifswald, Homburg, Kempten, Lingen [near Münster], Marburg, München, Radeberg [near Dresden], Rostock, Stuttgart, and Wiesbaden) and through the German TSC patient advocacy group (Tuberöse Sklerose Deutschland e.V., Wiesbaden, Germany). Paper questionnaires in German were sent to individuals with TSC between February and July 2019. Participants answered the questionnaire either alone or with caregiver support. In this analysis, we included only adult individuals ( $\geq 18$ years) who answered at least one of the main patient-reported outcome measures.

After receiving written informed consent from patients or their legal guardians (if applicable), we deemed all individuals with TSC that fulfilled the inclusion criteria as eligible. The diagnostic criteria for TSC were based on the latest recommendations established by the 2012 International TSC Consensus Conference [17]. We identified seven primary TSC manifestation categories such as epilepsy, structural brain defects, psychiatric, heart and circulatory system disorders, kidney and urinary tract disorders, dermatological system manifestations, respiratory system manifestations, and other manifestations [11]. We further identified frequent specific TSC manifestations within each primary category. Seizure 
and epilepsy syndrome classifications were adapted to the latest definitions established by the International League against Epilepsy $[18,19]$. We defined "active epilepsy" as experiencing at least one seizure within the 12 months preceding study participation [20]. This study received ethical approval and was registered with the German Clinical Trials Register (DRKS00016045; Universal Trial Number: U1111-1229-4714).

\section{Materials}

We asked individuals with TSC to complete a retrospective questionnaire based on their experiences during the previous 3 months. The questionnaire was successfully applied in earlier studies [21-25], and we adapted it for use in individuals with TSC. The questionnaire included the following instruments:

1) The EuroQoL-5 dimension-3 level inventory (EQ5D-3L) measures generic QoL. The EQ-5D comprises a visual analogue scale (VAS, range: $0-100$ units), with 100 representing the best and 0 representing the worst health imaginable. The EQ-5D contains 5 three-level Likert questions regarding mobility, self-care, usual activities, pain, and anxiety/depression. A single, continuous summary index is derived by transforming the EQ-5D Likert items with a "value set". This value set provides prespecified weights for each possible combination of answers according to preferences in a countryspecific population. The summary index score ranges from 1 (best imaginable health), 0 (health status equivalent to death) to negative values (health status "worse than death"). We derived summary index values using both the time-trade-off (TTO) and VAS methods, using the "eq5d" package (available from https://cran.r-project.org/web/ packages/eq5d/) for R (R Core Team, Vienna, Austria). We report the raw VAS scores alongside the EQ-5D summary index scores.

2) The Quality of Life in Epilepsy Inventory-31 items (QOLIE-31) measures health-related QoL (HRQoL) [26], which has been deemed appropriate for individuals with TSC [27]. The QOLIE-31 features 30 items across 7 dimensions (overall QoL, seizure worry, emotional well-being, energy/fatigue, cognitive function, medication effects, and social functioning) and a separate VAS analogous to the VAS in the EQ-5D. We calculated QOLIE-31 scores based on the manual (available from [28]). In brief, items in each of the 7 dimensions were first combined into subscale scores. A continuous overall score was obtained as a summary of the weighted subscores. The QOLIE-31 overall score ranges from 0 to 100 , with 100 representing the best overall disease-related health and 0 representing the worst health. The VAS is not included in the overall QOLIE-31 score but is reported separately.

3) The Neurological Disorders Depression Inventory for Epilepsy (NDDI-E) [29] is an externally validated instrument for assessing depression, developed for a cohort of epilepsy patients. The NDDI-E features 6 four-level Likert items regarding the frequency of common depressive symptoms, such as feeling guilty, frustrated, or weary of life. An aggregate value of $\geq 14$ points (range: $6-24$ points) is indicative of depressive mood in the validated German version [27].

4) The Epilepsy Stigma Scale measures disease-related stigma [28], using three questions ('I feel that some people are uncomfortable with me', 'I feel some people treat me like an inferior person,' and 'I feel some people would prefer to avoid me'). An affirmative answer to any question indicates stigma, with an aggregate value of 3 suggesting a severe stigma [27]. We dichotomised the Epilepsy Stigma Scale into 'severe stigma' and 'no or not severe stigma', according to Baker et al. [27].

5) We measured therapy-related adverse events with the Liverpool Adverse Events Profile (LAEP) inventory, which features 20 four-level Likert items using summarised item values (range 20-80). Based on previous cohorts, a cutoff score of 35 points was used to indicate relevant therapy-related adverse events [30].

6) We quantitatively assessed the TSC-related burden of illness using 20 four-level Likert items, each measuring the severity of burden from a possible TSC organ manifestation, and 33 additional questions regarding disease characteristics (e.g. genetics, affected organ systems, seizures, medications, and additional symptoms), healthcare resource use, and social situations.

In the German social system, individuals with disabilities who are entitled to certain monetary and social compensations are assigned a 'grade of disability', which quantifies the disability type and severity and determines compensation. The grade of disability is classified by an independent medical professional and ranges from 20 to 100 , in steps of 10 .

\section{Statistical analysis}

Descriptive analyses were conducted for sociodemographic and clinical characteristics. The following variables were analysed as potential QoL factors: age (median split for univariate analysis), sex (male/female), employment (yes/no), grade of disability $(\leq 80 />80)$, mutation type (TSC1/TSC2), active epilepsy (yes/no), 
structural central nervous system manifestation (yes/no), SEGA (yes/no), neuropsychiatric manifestation (yes/no), renal AML (yes/no), lymphangioleiomyomatosis (yes/ no), skin manifestation (yes/no), number of manifestations (quartile split for univariate analysis), anti-seizure medication polytherapy $(<2 \quad \mid \geq 2$ medications), use of everolimus (yes/no), and LAEP score $(<35 / \geq 35)$. Generic QoL (EQ-5D), HRQoL (QOLIE-31), and mood (NDDIE) results were compared relative to these potential factors using the Kruskal-Wallis-test, Chi-square test, and independent-samples $t$-test. The association between (HR) QoL and burden of illness and between HRQoL and LAEP score, number of organ manifestations, and grade of disability were assessed using correlation analysis (Pearson's correlation coefficient).

To evaluate potential predictors of HRQoL (dependent variable: QOLIE-31 overall score), we performed multiple linear regression analysis. All predictors defined as significant in the group comparisons that broadly overlapped with known predictors from the literature were included, and a non-hierarchical forced entry method was applied. We calculated effect sizes for significant individual predictors in the regression model based on the squares of the semi-partial predictor correlations $\left[r_{\text {part }}^{2} /\right.$ $\left(1-r_{\text {part }}^{2}\right)$, with cutoffs of $f^{2} \geq 0.02$ for small, $f^{2} \geq 0.15$ for medium, and $f^{2} \geq 0.35$ for large effect sizes, based on Cohen's suggestions [31]. According to power estimates by Miles and Shevlin [32], our sample size allowed for the detection of medium and large effect sizes in a fivepredictor model. We assessed the multicollinearity of all predictors and used tolerance as a measure of collinearity, with a threshold of 0.25 , below which collinearity was assumed. To assess predictors of severe diseaserelated stigma (dependent variable: Epilepsy Stigma Scale $=3$ vs fewer points), we used binomial logistic regression.

A $p$-value $<0.05$ (two-sided) was considered significant. We adjusted for multiple comparisons using the false-discovery rate method established by Benjamini and Hochberg [33], with a threshold for the falsediscovery rate of 0.05 . All $p$-values provided in the results section are significant after correction for multiple comparisons. In the tables, all $p$-values that remain significant after correction for multiple comparisons are marked with $(\dagger)$ and bold type. Statistical analysis was performed using IBM SPSS Statistics, version 26 (IBM Corp., Armonk, NY, USA) and R, version 3.6.2 (R Core Team, Vienna, Austria).

\section{Results}

\section{Demographic and clinical characteristics}

In total, 121 individuals were included in this analysis, with an average age of 31.0 years (standard deviation $[S D]=10.5$ years, range: $18-61$ years $)$, and $45.5 \%(n=55)$ were women. The median (interquartile range [IQR]) age at first TSC manifestation was during the first year of life (IQR: 0-3 years), and the median age of formal TSC diagnosis of TSC was 2.5 years (IQR: 0-14.3 years). Individuals had a median of 5 TSC manifestations (range: 1-8). The most commonly affected organ systems were the skin $(95.9 \%, n=116)$ and brain, including epilepsy/seizures $(76.9 \%, n=93)$ and structural brain manifestations $(71.1 \%, n=86)$. The most common specific manifestations were facial angiofibroma $(83.5 \%, n=$ $101)$, seizures $(76.9 \%, n=93)$, renal AML $(57.9 \%, n=70)$, hypomelanotic macules $(56.2 \%, n=68)$, and cortical tubers $(52.9 \%, n=64)$. Neuropsychiatric manifestations were reported by 60 (49.6\%) respondents. Among individuals with seizures, $53.8 \%(n=50)$ had active epilepsy, indicating that they experienced at least one seizure during the 12 months preceding the date of study participation. Almost half of the study population $(46.3 \%, n=56)$ reported $\geq 12$ years of formal education. Of the 83 individuals that disclosed employment status, $41.0 \%(n=34)$ were currently unemployed. Additional clinical and demographic information is provided in Table 1.

\section{Generic and health-related quality of life, mood, and stigma \\ $E Q-5 D$}

For the entire study population, the EQ-5D TTO summary index score was $0.818(S D=0.262$, range: -0.140 to 1, Table 2). Generic QoL was lower among unemployed (mean $=0.661, S D=0.341)$ than employed individuals $\quad($ mean $=0.920, S D=0.115 ; p<0.001)$, and lower among those with a disability grade above 80 (mean $=0.681, S D=0.318)$ compared with those with lower grades $($ mean $=0.916, S D=0.101 ; p<0.001)$. QoL did not differ between sex and age categories. QoL was worse among those with active epilepsy (mean $=0.729$, $S D=0.305)$ than in those who were seizure-free over the past 12 months (mean $=0.883, S D=0.205 ; p=0.001$ ). Those with neuropsychiatric manifestations had significantly worse QoL than those without (mean $=0.693$, $S D=0.314$ vs mean $=0.938, S D=0.107 ; p<0.001$ ). Those with higher numbers of manifestations reported worse QoL than those less affected by TSC (1-3 manifestations: mean $=0.931, S D=0.159 ; 4-6$ manifestations mean $=0.805, S D=0.264 ; 7-8$ manifestations: mean $=$ $0.662, S D=0.330 ; p=0.001$; Table 2). QoL did not differ significantly between those with TSC1 and TSC2 variations. The presence of renal AML, skin manifestations, structural brain manifestations (specifically SEGA), and lung manifestations (lymphangioleiomyomatosis) were not associated with worse generic QoL. Only 12 individuals reported the presence of lymphangioleiomyomatosis. Anti-seizure medication polytherapy was not associated with a worse generic QoL, nor was the use of 
Table 1 Sociodemographic and clinical characteristics of participants $(n=121)$

\begin{tabular}{|c|c|c|}
\hline & & All patients, $n=121$ \\
\hline \multirow[t]{4}{*}{ Age, years } & Mean $\pm S D$ & $31.0 \pm 10.5$ \\
\hline & Range & $18-61$ \\
\hline & Median & 29.0 \\
\hline & IQR & $22-37$ \\
\hline \multirow[t]{2}{*}{ Sex, $n(\%)$} & Female & $55(45.5)$ \\
\hline & Male & $66(55.5)$ \\
\hline \multirow[t]{4}{*}{ Age at first manifestation of TSC, years } & Mean $\pm S D$ & $3.8 \pm 8.1$ \\
\hline & Range & $0-41$ \\
\hline & Median & 0 \\
\hline & IQR & $0-3$ \\
\hline \multirow[t]{4}{*}{ Age at TSC diagnosis, years } & Mean $\pm S D$ & $8.9 \pm 13.0$ \\
\hline & Range & $0-53$ \\
\hline & Median & 2.5 \\
\hline & IQR & $0-14.3$ \\
\hline \multirow[t]{7}{*}{ First TSC symptom/sign, n (\%) } & Epilepsy/seizures & $65(53.7)$ \\
\hline & Skin manifestation & $24(19.8)$ \\
\hline & Cardiac rhabdomyoma & $7(5.8)$ \\
\hline & Renal manifestation & $4(3.3)$ \\
\hline & Eye manifestation & $3(2.5)$ \\
\hline & Cardiac arrhythmia & $3(2.5)$ \\
\hline & Other & $12(9.9)$ \\
\hline \multirow[t]{7}{*}{ Genetic testing, $n(\%)$} & Yes, TSC1 & $25(20.7)$ \\
\hline & Yes, TSC2 & $31(25.6)$ \\
\hline & Yes, TSC2/PKD1 contiguous gene & $1(0.8)$ \\
\hline & Yes, but no mutation identified (NMI) & $16(13.2)$ \\
\hline & Yes, mutation not specified & $10(8.3)$ \\
\hline & No genetic testing & $30(24.8)$ \\
\hline & Unknown & $8(6.6)$ \\
\hline \multirow[t]{16}{*}{ Clinical manifestations, $n$ (\%) } & Skin & $116(95.9)$ \\
\hline & Angiofibroma & $101(83.5)$ \\
\hline & Hypomelanotic macules & $68(56.2)$ \\
\hline & Shagreen patches & $59(48.8)$ \\
\hline & Ungual fibromas & $13(10.7)$ \\
\hline & Skin tags & $4(3.3)$ \\
\hline & Café au lait-spots & $4(3.3)$ \\
\hline & Epilepsy/seizures & $93(76.9)$ \\
\hline & Active epilepsy & $50(41.3)$ \\
\hline & Brain structural & $86(71.1)$ \\
\hline & Tuber & $64(52.9)$ \\
\hline & SEGA & $51(42.1)$ \\
\hline & Hydrocephalus & $3(2.5)$ \\
\hline & Kidney & $85(70.2)$ \\
\hline & AML & $70(57.9)$ \\
\hline & Heart & $62(51.2)$ \\
\hline
\end{tabular}


Table 1 Sociodemographic and clinical characteristics of participants $(n=121)$ (Continued)

\begin{tabular}{|c|c|c|}
\hline & & All patients, $n=121$ \\
\hline & Cardiac rhabdomyoma & $29(24.0)$ \\
\hline & Neuropsychiatric & $60(49.6)$ \\
\hline & Lymphangioleiomyomatosis & $12(9.9)$ \\
\hline & Other & $50(41.3)$ \\
\hline \multirow[t]{2}{*}{ Number of manifestations } & Median & 5 \\
\hline & Range & $1-8$ \\
\hline \multirow[t]{6}{*}{ Highest school graduation certificate, $n(\%)$} & High school ('Abitur/Fachabitur') & $29(24.0)$ \\
\hline & Intermediate school ('Realschulabschluss/mittlere Reife') & $27(22.3)$ \\
\hline & Secondary modern school ('Hauptschulabschluss') & $10(8.3)$ \\
\hline & Still in school & $6(5.0)$ \\
\hline & No certificate & $29(24.0)$ \\
\hline & Other & $19(15.7)$ \\
\hline \multirow[t]{5}{*}{ Current occupation, $n$ (\%) } & Employee full-time & $35(28.9)$ \\
\hline & Unemployment or unable to work due to TSC & $29(23.4)$ \\
\hline & Employee part-time & $12(9.9)$ \\
\hline & Unemployment or unable to work due to other illness & $5(4.1)$ \\
\hline & Self-employed & $2(1.7)$ \\
\hline
\end{tabular}

$A M L$ angiomyolipoma, IQR interquartile range, $P K D$ polycystic kidney disease, SEGA subependymal giant cell astrocytoma, SD standard deviation, $T S C$ tuberous sclerosis complex

everolimus (in the TTO index). However, those with a LAEP score $\geq 35$ points, indicating moderate-to-severe therapy-related adverse events, had significantly worse QoL than those with milder therapy-related adverse events (mean $=0.757, S D=0.287$ vs mean $=0.960, S D=$ $0.093 ; p<0.001)$. The EQ-5D VAS summary index demonstrated significant differences for the same variables as the TTO summary index, plus everolimus use. Individuals who used everolimus reported worse QoL than those without everolimus use in the VAS $(p=0.007)$ but not the TTO index $(p=0.141)$. Using individual EQ-5D subscales, the highest frequency of individuals reporting any degree of problems was identified in the usual care (47.5\%) and anxiety/depression domains (40.5\%), followed by self-care $(37.4 \%)$, pain $(27.0 \%)$, and mobility (12.4\%).

Visual analysis of a histogram displaying both the TTOand VAS-transformed EQ-5D summary index scores for the whole study population identified a highly rightskewed distribution. Further comparison of the QOLIE-31 overall scores and the EQ-5D summary indices demonstrated a large share of maximum EQ-5D summary index values among individuals with QOLIE-31 overall scores down to about 62.5 , indicating a substantial ceiling effect for the EQ-5D instrument in our cohort.

\section{QOLIE-31}

For the overall study population, the QOLIE-31 overall score was $63.5(S D=19.7$, range: $12.15-100$; Table 3$)$.
The sociodemographic, clinical, and therapy-related variables associated with a worse HRQoL were generally identical to those identified by the EQ-5D summary index score analysis. The QOLIE-31 overall score was lower among unemployed than employed individuals $($ mean $=51.5, S D=16.5$ vs mean $=71.7, S D=19.9 ; p<$ 0.001 ) and among those with a disability grade above 80 compared with those with lower grades (mean $=54.2$, $S D=16.5$ vs mean $=66.1, S D=16.5 ; p<0.001)$. Scores were lower in those with active epilepsy than in 12month seizure-free individuals (mean $=52.1, S D=17.7$ vs mean $=71.7, S D=16.7 ; p<0.001$ ) and in those with neuropsychiatric manifestations compared with those without (mean $=53.2, S D=18.0$ vs. mean $=73.8, S D=$ $15.4 ; p<0.001)$. Those with $1-3$ TSC manifestations had better HRQoL than those with 4-6 or 7-8 manifestations $($ mean $=71.7, S D=18.4$ vs mean $=62.8, S D=18.9$ vs mean $=52.9, S D=20.0 ; p=0.007$; Table 3 ). Those with structural brain manifestation (not specifically SEGA) had worse HRQoL than those without (mean = $60.5, S D=20.1$ vs mean $=71.5, S D=16.3, p=0.007$ ) but only for the overall score, not the VAS $(p=0.232)$. No other clinical aspects significantly influenced HRQoL. Those with a LAEP of $\geq 35$ points had significantly worse HRQoL than those more mildly affected (mean $=56.0$, $S D=17.2$ vs mean $=80.5, S D=12.7 ; p<0.001)$.

The number of manifestations $(r=-0.35$, 95\% confidence interval $(\mathrm{CI}):-0.49$ to $-0.18, p<0.001)$, grade of disability $(r=-0.38,95 \% \mathrm{CI}:-0.54$ to $-0.19, p<0.001)$ 
Table 2 Comparisons of generic quality of life among individuals with TSC, as measured by the EQ-5D (German version) questionnaire, according to several potential predictors and assessed by the Kruskal-Wallis and Chi-square tests

\begin{tabular}{|c|c|c|c|c|c|c|c|}
\hline Predictor & Measure & $N$ & Category & Mean & $\pm S D$ & $95 \% \mathrm{Cl}$ & $p$-value* \\
\hline \multicolumn{8}{|l|}{ Sociodemographic aspects } \\
\hline \multirow[t]{4}{*}{ Sex } & VAS & 64 & Male & 67.9 & 19.7 & $63.0-72.9$ & 0.768 \\
\hline & & 54 & Female & 66.7 & 21.2 & $60.9-72.5$ & \\
\hline & Index (TTO) & 64 & Male & 0.796 & 0.267 & $0.730-0.863$ & 0.320 \\
\hline & & 54 & Female & 0.843 & 0.257 & $0.773-0.914$ & \\
\hline \multirow[t]{4}{*}{ Age } & VAS & 54 & $18-28$ y & 72.0 & 20.4 & $66.4-77.6$ & 0.020 \\
\hline & & 62 & $29-61$ y & 68.2 & 19.6 & $58.3-68.2$ & \\
\hline & Index (TTO) & 54 & $18-28$ y & 0.834 & 0.238 & $0.769-0.898$ & 0.309 \\
\hline & & 62 & $29-61$ y & 0.800 & 0.286 & $0.727-0.873$ & \\
\hline \multirow[t]{4}{*}{ Employment } & VAS & 49 & Yes & 70.8 & 18.2 & $65.6-76.0$ & 0.017 \\
\hline & & 33 & No & 58.7 & 21.7 & $51.0-66.4$ & \\
\hline & Index (TTO) & 49 & Yes & 0.920 & 0.115 & $0.887-0.953$ & $<0.001 \dagger$ \\
\hline & & 33 & No & 0.661 & 0.341 & $0.540-0.782$ & \\
\hline \multirow[t]{4}{*}{ Grade of disability } & VAS & 36 & $0-80$ & 72.0 & 15.9 & $66.6-77.4$ & $0.001 \dagger$ \\
\hline & & 56 & $90-100$ & 58.6 & 20.3 & $53.1-64.0$ & \\
\hline & Index (TTO) & 36 & $0-80$ & 0.916 & 0.101 & $0.882-0.950$ & $<0.001 \dagger$ \\
\hline & & 56 & $90-100$ & 0.681 & 0.318 & $0.596-0.766$ & \\
\hline \multicolumn{8}{|l|}{ Clinical aspects } \\
\hline \multirow[t]{4}{*}{ Mutation type } & VAS & 24 & TSC1 & 72.8 & 18.5 & $65.0-80.6$ & 0.198 \\
\hline & & 30 & TSC2 & 65.0 & 24.2 & $56.0-74.1$ & \\
\hline & Index (TTO) & 24 & TSC1 & 0.803 & 0.288 & $0.682-0.924$ & 0.662 \\
\hline & & 30 & TSC2 & 0.793 & 0.296 & $0.682-0.904$ & \\
\hline \multirow[t]{4}{*}{ Active epilepsy } & VAS & 50 & Yes & 60.0 & 20.1 & $54.3-65.7$ & $0.001 \dagger$ \\
\hline & & 71 & $\mathrm{Sz}$ freedom $>12 \mathrm{~m}$ & 72.3 & 18.9 & $68.2-77.4$ & \\
\hline & Index (TTO) & 50 & Yes & 0.729 & 0.305 & $0.642-0.816$ & $0.001 \dagger$ \\
\hline & & 71 & Sz freedom $>12 \mathrm{~m}$ & 0.883 & 0.205 & $0.834-0.939$ & \\
\hline \multirow[t]{4}{*}{ Structural brain } & VAS & 83 & Yes & 65.4 & 20.3 & $61.0-69.9$ & 0.116 \\
\hline & & 35 & No & 72.0 & 20.0 & $65.1-78.8$ & \\
\hline & Index (TTO) & 83 & Yes & 0.793 & 0.281 & $0.732-0.855$ & 0.123 \\
\hline & & 35 & No & 0.876 & 0.206 & $0.805-0.947$ & \\
\hline \multirow[t]{4}{*}{ SEGA } & VAS & 50 & Yes & 62.2 & 20.5 & $56.3-68.0$ & 0.017 \\
\hline & & 68 & No & 71.2 & 19.5 & $66.5-75.9$ & \\
\hline & Index (TTO) & 50 & Yes & 0.802 & 0.268 & $0.725-0.877$ & 0.597 \\
\hline & & 68 & No & 0.830 & 0.260 & $0.767-0.893$ & \\
\hline \multirow[t]{4}{*}{ Neuropsychiatric } & VAS & 60 & Yes & 59.1 & 21.4 & $53.5-64.7$ & $<0.001 \dagger$ \\
\hline & & 61 & No & 75.4 & 15.7 & $71.3-75.4$ & \\
\hline & Index (TTO) & 60 & Yes & 0.693 & 0.314 & $0.610-0.776$ & $<0.001 \dagger$ \\
\hline & & 61 & No & 0.938 & 0.107 & $0.911-0.966$ & \\
\hline \multirow[t]{4}{*}{ AML } & VAS & 69 & Yes & 65.9 & 21.2 & $60.8-71.0$ & 0.317 \\
\hline & & 49 & No & 69.4 & 19.2 & $63.9-74.9$ & \\
\hline & Index (TTO) & 70 & Yes & 0.805 & 0.276 & $0.739-0.872$ & 0.979 \\
\hline & & 51 & No & 0.835 & 0.244 & $0.765-0.906$ & \\
\hline Lymphangioleio-myomatosis & VAS & 12 & Yes & 67.5 & 12.7 & $59.4-75.6$ & 0.844 \\
\hline
\end{tabular}


Table 2 Comparisons of generic quality of life among individuals with TSC, as measured by the EQ-5D (German version) questionnaire, according to several potential predictors and assessed by the Kruskal-Wallis and Chi-square tests (Continued)

\begin{tabular}{|c|c|c|c|c|c|c|c|c|}
\hline Predictor & Measure & $N$ & Category & Mean & $\pm S D$ & $95 \% \mathrm{Cl}$ & $p$-value* & \\
\hline & & 106 & No & 67.4 & 21.1 & $63.3-71.4$ & & \\
\hline & Index (TTO) & 12 & Yes & 0.819 & 0.320 & $0.615-1.023$ & 0.839 & \\
\hline & & 106 & No & 0.818 & 0.257 & $0.768-0.867$ & & \\
\hline \multirow[t]{4}{*}{ Skin } & VAS & 114 & Yes & 67.1 & 20.5 & $63.3-70.9$ & 0.469 & \\
\hline & & 4 & No & 75.0 & 13.5 & $63.3-96.6$ & & \\
\hline & Index (TTO) & 116 & Yes & 0.815 & 0.267 & $0.767-0.865$ & 0.504 & \\
\hline & & 5 & No & 0.890 & 0.087 & $0.753-1.028$ & & \\
\hline \multirow[t]{6}{*}{ Number of affected organs } & VAS & 30 & $1-3$ & 80.9 & 12.3 & $76.3-85.5$ & ref. & $<0.001 \dagger$ \\
\hline & & 72 & $4-6$ & 64.5 & 20.3 & $59.7-69.3$ & 0.005 & \\
\hline & & 16 & $7-8$ & 55.1 & 20.3 & $44.2-65.9$ & 0.048 & \\
\hline & Index (TTO) & 30 & $1-3$ & 0.931 & 0.159 & $0.872-0.990$ & ref. & $0.001 \dagger$ \\
\hline & & 72 & $4-6$ & 0.805 & 0.264 & $0.743-0.867$ & 0.037 & \\
\hline & & 16 & $7-8$ & 0.662 & 0.330 & $0.487-0.838$ & 0.009 & \\
\hline \multicolumn{9}{|l|}{ Therapeutic aspects } \\
\hline \multirow[t]{4}{*}{ Anti-seizure medication polytherapy ${ }^{a}$} & VAS & 53 & Yes & 62.3 & 20.2 & $61.7-74.9$ & 0.223 & \\
\hline & & 37 & No & 68.3 & 19.8 & $57.3-68.4$ & & \\
\hline & Index (TTO) & 53 & Yes & 0.759 & 0.282 & $0.682-0.837$ & 0.081 & \\
\hline & & 40 & No & 0.851 & 0.250 & $0.767-0.934$ & & \\
\hline \multirow[t]{4}{*}{ Everolimus } & VAS & 50 & Yes & 61.6 & 20.3 & $55.8-67.4$ & $0.007 \dagger$ & \\
\hline & & 68 & No & 71.6 & 19.4 & $66.9-76.3$ & & \\
\hline & Index (TTO) & 50 & Yes & 0.772 & 0.286 & $0.691-0.854$ & 0.141 & \\
\hline & & 68 & No & 0.851 & 0.241 & $0.793-0.910$ & & \\
\hline \multirow[t]{4}{*}{ LAEP } & VAS & 34 & $<35$ & 62.6 & 19.7 & $58.3-66.9$ & $<0.001 \dagger$ & \\
\hline & & 83 & $\geq 35$ & 78.4 & 17.5 & $72.3-84.5$ & & \\
\hline & Index (TTO) & 34 & $<35$ & 0.757 & 0.287 & $0.695-0.820$ & $<0.001 \dagger$ & \\
\hline & & 83 & $\geq 35$ & 0.960 & 0.093 & $0.928-0.993$ & & \\
\hline
\end{tabular}

$A M L$ angiomyolipoma, $C l$ confidence interval, EQ-5D EuroQoL-5 dimensions, TTO time-trade-off method, VAS visual analogue scale, LAEP Liverpool Adverse Events Profile, $m$ months, QOLIE-31 Quality of Life in Epilepsy Inventory-31 items, ref. reference category, SD standard deviation, SEGA subependymal giant cell astrocytoma, sz seizure, TSC tuberous sclerosis complex, $y$ years

ancludes only individuals with TSC-associated epilepsy/seizures

${ }^{*}$ Comparisons corrected for multiple testing using the Benjamini-Hochberg false-discovery rate method, $\dagger$ and bold type denotes a $q$-value of $<0.05$ (false discovery rate)

and LAEP score $(r=-0.76,95 \% \mathrm{CI}:-0.83$ t o $-0.67, p<$ 0.001 ) were each significantly correlated with the QOLIE-31 overall score (Fig. 1).

\section{NDDI-e}

The mean NDDI-E value in our cohort was 12.4 (median $=12$, range: $6-23$ ), only slightly below the cutoff of 14 points for probable depression. Several variables were associated with worse NDDI-E values (the presence of structural brain or neuropsychiatric manifestations, a higher number of affected organs, and LAEP $\geq 35$ points). However, only the subgroup with the most TSC manifestations had a mean NDDI-E greater than 14 points. The median reached 14 points in those with at least six TSC manifestations, indicating that at least 50\% of these severely affected individuals are likely to show depression symptoms (Table 4).

\section{Epilepsy stigma scale}

Severe stigma (Epilepsy Stigma Scale $=3$ points) was associated with neuropsychiatric manifestations $(p=$ $0.004)$, epilepsy $(p=0.011$, but not active epilepsy $[p=$ $0.145])$, structural brain manifestations $(p=0.029)$, SEGA $(p=0.029)$, and worse LAEP scores $(p<0.001)$. In binomial logistic regression, only the LAEP score independently predicted severe stigma, with each point increase in LAEP score increasing the odds of perceiving a severe stigma by $7 \%$ (estimate: 0.067 , standard error: $0.02, p=0.001)$. 
Table 3 Comparisons of health-related quality of life among individuals with TSC, as measured by the QOLIE-31 (German version) questionnaire, according to several potential predictors and assessed using the Kruskal-Wallis and Chi-square tests

\begin{tabular}{|c|c|c|c|c|c|c|c|}
\hline Predictor & Measure & $N$ & Category & Mean & $\pm S D$ & $95 \% \mathrm{Cl}$ & $p$-value* \\
\hline \multicolumn{8}{|l|}{ Sociodemographic aspects } \\
\hline \multirow[t]{4}{*}{ Sex } & VAS & 65 & Male & 64.2 & 19.9 & $59.2-69.1$ & 0.790 \\
\hline & & 55 & Female & 63.6 & 22.1 & $57.6-69.5$ & \\
\hline & Overall score & 65 & Male & 62.2 & 20.2 & $57.5-67.2$ & 0.277 \\
\hline & & 55 & Female & 65.5 & 19.0 & $60.3-70.6$ & \\
\hline \multirow[t]{4}{*}{ Age } & VAS & 55 & $18-28$ y & 67.1 & 20.3 & $61.6-72.6$ & 0.136 \\
\hline & & 63 & $29-61$ y & 60.9 & 21.0 & $55.6-66.2$ & \\
\hline & Overall score & 55 & $18-28$ y & 64.4 & 20.6 & $58.9-70.0$ & 0.666 \\
\hline & & 53 & $29-61$ y & 62.7 & 19.0 & $57.9-67.5$ & \\
\hline \multirow[t]{4}{*}{ Employment } & VAS & 49 & Yes & 70.6 & 19.7 & $64.9-76.2$ & $0.001 \dagger$ \\
\hline & & 34 & No & 54.7 & 20.8 & $46.4-60.9$ & \\
\hline & Overall score & 49 & Yes & 71.7 & 19.9 & $66.9-76.4$ & $<0.001 \dagger$ \\
\hline & & 34 & No & 51.5 & 16.5 & $44.5-58.4$ & \\
\hline \multirow[t]{4}{*}{ Grade of disability } & VAS & 38 & $0-80$ & 65.0 & 17.4 & $59.2-70.7$ & $0.008+$ \\
\hline & & 56 & $90-100$ & 54.3 & 18.1 & $49.4-59.1$ & \\
\hline & Overall score & 38 & $0-80$ & 66.1 & 16.5 & $60.7-71.5$ & $<0.001 \dagger$ \\
\hline & & 56 & $90-100$ & 54.2 & 16.5 & $49.7-58.6$ & \\
\hline \multicolumn{8}{|l|}{ Clinical aspects } \\
\hline \multirow[t]{4}{*}{ Mutation type } & VAS & 25 & TSC1 & 70.3 & 18.0 & $62.9-77.8$ & 0.099 \\
\hline & & 31 & TSC2 & 61.0 & 23.5 & $52.3-69.6$ & \\
\hline & Overall score & 25 & TSC1 & 68.8 & 17.3 & $61.6-75.9$ & 0.424 \\
\hline & & 31 & TSC2 & 63.0 & 21.4 & $55.2-70.9$ & \\
\hline \multirow[t]{4}{*}{ Active epilepsy } & VAS & 49 & Yes & 56.7 & 19.4 & $51.2-62.3$ & $0.003+$ \\
\hline & & 71 & No & 68.8 & 20.5 & $64.0-73.7$ & \\
\hline & Overall score & 49 & Yes & 52.1 & 17.7 & $47.0-57.2$ & $<0.001 \dagger$ \\
\hline & & 71 & No & 71.7 & 16.7 & $67.8-75.7$ & \\
\hline \multirow[t]{4}{*}{ Structural brain } & VAS & 85 & Yes & 62.3 & 20.9 & $57.8-66.8$ & 0.232 \\
\hline & & 35 & No & 67.7 & 20.5 & $60.6-74.7$ & \\
\hline & Overall score & 85 & Yes & 60.5 & 20.1 & $56.2-64.8$ & $0.007+$ \\
\hline & & 35 & No & 71.5 & 16.3 & $65.9-77.1$ & \\
\hline \multirow[t]{4}{*}{ SEGA } & VAS & 50 & Yes & 62.0 & 22.1 & $55.7-68.3$ & 0.458 \\
\hline & & 70 & No & 65.2 & 20.0 & $60.5-70.0$ & \\
\hline & Overall score & 50 & Yes & 59.4 & 20.1 & $53.7-65.2$ & 0.041 \\
\hline & & 70 & No & 66.7 & 18.9 & $62.2-71.2$ & \\
\hline \multirow[t]{4}{*}{ Neuropsychiatric } & VAS & 59 & Yes & 55.1 & 20.4 & $49.8-60.5$ & $<0.001 \dagger$ \\
\hline & & 61 & No & 72.3 & 17.6 & $67.8-76.9$ & \\
\hline & Overall score & 59 & Yes & 53.2 & 18.0 & $48.5-57.9$ & $<0.001 \dagger$ \\
\hline & & 61 & No & 73.8 & 15.4 & $69.9-77.8$ & \\
\hline \multirow[t]{4}{*}{ AML } & VAS & 70 & Yes & 61.5 & 22.3 & $56.2-66.9$ & 0.146 \\
\hline & & 50 & No & 67.2 & 18.4 & $61.9-72.4$ & \\
\hline & Overall score & 70 & Yes & 63.6 & 20.8 & $58.6-68.5$ & 0.819 \\
\hline & & 50 & No & 63.9 & 18.2 & $58.7-69.0$ & \\
\hline Lymphangioleiomyomatosis & VAS & 12 & Yes & 63.3 & 14.4 & $54.2-72.5$ & 0.829 \\
\hline
\end{tabular}


Table 3 Comparisons of health-related quality of life among individuals with TSC, as measured by the QOLIE-31 (German version) questionnaire, according to several potential predictors and assessed using the Kruskal-Wallis and Chi-square tests (Continued)

\begin{tabular}{|c|c|c|c|c|c|c|c|c|}
\hline Predictor & Measure & $N$ & Category & Mean & $\pm S D$ & $95 \% \mathrm{Cl}$ & $p$-value* & \\
\hline & & 108 & No & 63.9 & 21.5 & $59.8-68.1$ & & \\
\hline & Overall score & 12 & Yes & 70.2 & 14.9 & $60.8-79.7$ & 0.256 & \\
\hline & & 108 & No & 63.0 & 20.0 & $59.2-66.8$ & & \\
\hline \multirow[t]{4}{*}{ Skin } & VAS & 115 & Yes & 63.8 & 20.9 & $59.9-67.7$ & 0.822 & \\
\hline & & 5 & No & 66.0 & 20.7 & $40.3-91.8$ & & \\
\hline & Overall score & 115 & Yes & 63.7 & 19.6 & $60.1-67.3$ & 0.855 & \\
\hline & & 5 & No & 63.3 & 22.9 & $34.8-91.8$ & & \\
\hline \multirow[t]{6}{*}{ Number of affected organs } & VAS & 31 & $1-3$ & 76.7 & 16.7 & $70.6-82.8$ & Ref & $<0.001 \dagger$ \\
\hline & & 72 & $4-6$ & 62.2 & 19.2 & $57.7-66.7$ & 0.001 & \\
\hline & & 17 & $7-8$ & 47.7 & 21.6 & $36.6-58.8$ & $<0.001$ & \\
\hline & Overall score & 31 & $1-3$ & 71.7 & 18.4 & $65.0-78.5$ & Ref & $0.007 \dagger$ \\
\hline & & 72 & $4-6$ & 62.8 & 18.9 & $58.4-67.2$ & 0.026 & \\
\hline & & 17 & $7-8$ & 52.9 & 20.0 & $42.6-63.2$ & 0.002 & \\
\hline \multicolumn{9}{|l|}{ Therapeutic aspects } \\
\hline \multirow[t]{4}{*}{ Anti-seizure medication polytherapy ${ }^{a}$} & VAS & 52 & Yes & 58.1 & 19.0 & $52.8-64.4$ & 0.178 & \\
\hline & & 31 & No & 64.8 & 20.1 & $57.4-72.2$ & & \\
\hline & Overall score & 52 & Yes & 54.9 & 17.8 & $49.9-59.8$ & 0.032 & \\
\hline & & 31 & No & 63.5 & 18.6 & $56.6-70.3$ & & \\
\hline \multirow[t]{4}{*}{ Everolimus } & VAS & 51 & Yes & 59.3 & 22.0 & $53.1-65.5$ & 0.063 & \\
\hline & & 69 & No & 67.3 & 19.4 & $62.6-71.9$ & & \\
\hline & Overall score & 51 & Yes & 60.5 & 20.0 & $54.9-66.1$ & 0.212 & \\
\hline & & 69 & No & 66.1 & 19.1 & $61.5-70.7$ & & \\
\hline \multirow[t]{4}{*}{ LAEP } & VAS & 36 & $<35$ & 76.7 & 18.0 & $70.6-82.8$ & $<0.001 \dagger$ & \\
\hline & & 83 & $\geq 35$ & 58.0 & 19.3 & $53.8-62.2$ & & \\
\hline & Overall score & 36 & $<35$ & 80.5 & 12.7 & $76.2-84.7$ & $<0.001 \dagger$ & \\
\hline & & 83 & $\geq 35$ & 56.0 & 17.2 & $52.3-59.8$ & & \\
\hline
\end{tabular}

$A M L$ angiomyolipoma, CI confidence interval, LAEP Liverpool Adverse Events Profile, QOLIE-31 Quality of Life in Epilepsy Inventory-31 items, ref. reference category, SD standard deviation, SEGA subependymal giant cell astrocytoma, TSC tuberous sclerosis complex, VAS visual analogue scale, y years ancludes only individuals with TSC-associated epilepsy/seizures

*Comparisons corrected for multiple testing using the Benjamini-Hochberg false-discovery rate method, $\uparrow$ and bold type denotes a $q$-value of $<0.05$

(false-discovery rate)

Association between quality of life and burden of illness Both generic QoL and HRQoL were highly correlated with a 20-item burden of illness index (item sum/number of noted items). The EQ-5D correlated negatively with the burden of illness index $(r=-0.53,95 \% \mathrm{CI}$ : 0.65 to $-0.39, p<0.001$ ), indicating that higher disease burden was associated with worse generic QoL. The HRQoL was similarly correlated with increasing burden of illness $(r=-0.53,95 \% \mathrm{CI}:-0.65$ to $-0.39, p<0.001$; Fig. 1).

\section{Comparison of (health-related) quality of life between disorders}

The HRQoL-VAS was lower among our active epilepsy cohort than among a cohort of 60 patients with drugrefractory epilepsy [34] (Fig. 2a; $p<0.001, t$-test).
Normative values exist for the QOLIE-31 subscales, allowing external comparisons based on $T$-scores $(T=50$ represents the average value for each subscale) [35]. We found a mean "seizure worry" score of $67.0(S D=31.7)$ among our 93 individuals with TSC-associated epilepsy, indicating a slightly lower seizure worry than average $(T=53)$ [36] and compared with 24 patients of a large registry of individuals with TSC (TuberOus SClerosis registry to increase disease Awareness [TOSCA]) [16] (Fig. 2b, $p<0.001, t$-test). The overall QoL subscale value in our TSC-epilepsy cohort was slightly below average (mean $=62.9, S D=18.8, T=48$ ) but better than that in the comparator registry $(p=0.0128, t$-test). Emotional well-being was slightly below average in our cohort (mean $=61.9, S D=20.0, T=47$ ) but better than that in the TOSCA registry $(p=0.0321, t$-test $)$. Fatigue 


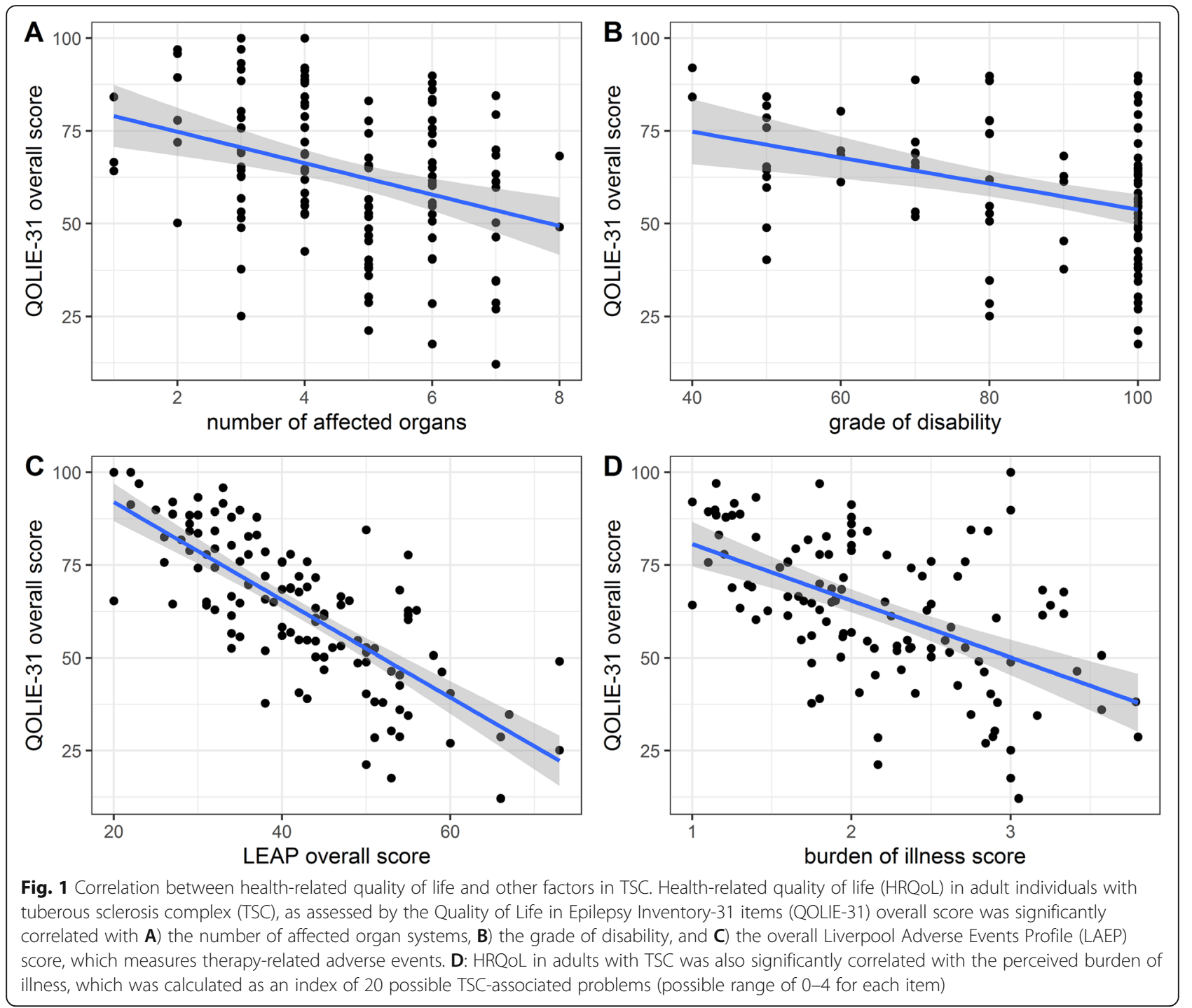

(mean $=50.7, S D=18.4)$, cognitive functioning $($ mean $=$ $54.7, S D=24.7$ ), and social functioning (mean $=58.8$, $S D=28.5$ ) in our patients were all slightly below average for an epilepsy cohort $(T=47)$; however, fatigue was higher in our active epilepsy subgroup than in drugrefractory medial temporal lobe epilepsy patients [34] (Fig. 2a, $p<0.001, t$-test). Medication-associated adverse effects were average compared with an epilepsy cohort (mean $=65.7, S D=24.2, T=53$ ) but lower than in the comparator registry $(p=0.0138, t$-test). Figure 2 shows additional non-significant differences between our cohort and the drug-refractory temporal lobe epilepsy and TOSCA cohorts.

Mildly affected ( $\leq 3$ manifestations) individuals report a generic QoL of 80.9 ( $S D=12.3$, corresponding EQ-5D TTO index: $0.931, S D=0.159)$, comparable to or even better than the overall German population with a single health issue (72.3 according to [37]). More severe manifestations were associated with significantly worse QoL (EQ-5D TTO index: 0.784, SD =0.279), similar to those with severe chronic diseases, including severe episodic migraines with more than eight headache days per month (EQ-5D index: 0.77, $S D=0.24$; Fig. 3) [38].

\section{Predictors of health-related quality of life}

In multiple linear regression analysis, the overall LAEP score was an independent predictor of worse HRQoL, with each additional LAEP score point reducing overall QOLIE-31 by almost one point $(\mathrm{B}=-0.906,95 \% \mathrm{CI}$ : 1.192 to $-0.621, p<0.001)$, with large effect size $\left(f^{2}=\right.$ $0.71)$. The presence of active epilepsy reduces the QOLIE-31 score by 12.6 points $(\mathrm{B}=-12.608,95 \% \mathrm{CI}$ : 18.234 to $-6.981, p<0.001)$, with large effect size $\left(f^{2}=\right.$ $0.35)$. The presence of neuropsychiatric manifestations worsens QOLIE-31 by 10.4 points $(\mathrm{B}=-10.401,95 \% \mathrm{CI}$ : -17.034 to $-3.769, p=0.003)$, with medium effect size 
Table 4 Comparisons of mood among individuals with TSC, as measured by the NDDI-E (German version) questionnaire, according to several potential predictors and assessed using Kruskal-Wallis and Chi-square tests

\begin{tabular}{|c|c|c|c|c|c|c|}
\hline Predictor & $N$ & Category & Mean & $\pm S D$ & $95 \% \mathrm{Cl}$ & $p$-value* \\
\hline \multicolumn{7}{|l|}{ Sociodemographic aspects } \\
\hline \multirow[t]{2}{*}{ Sex } & 66 & Male & 12.32 & 4.0 & $11.3-13.3$ & 0.767 \\
\hline & 55 & Female & 12.58 & 4.2 & $11.5-13.7$ & \\
\hline \multirow[t]{2}{*}{ Age } & 56 & $18-28$ y & 11.8 & 4.0 & $10.8-12.9$ & 0.192 \\
\hline & 63 & $29-61$ y & 13.0 & 4.2 & $11.9-14.0$ & \\
\hline \multirow[t]{2}{*}{ Employment } & 49 & Yes & 11.8 & 3.9 & $10.7-12.9$ & 0.056 \\
\hline & 34 & No & 13.8 & 4.6 & $12.2-15.9$ & \\
\hline \multirow[t]{2}{*}{ Grade of disability } & 38 & $0-80$ & 12.5 & 4.1 & $11.2-13.9$ & 0.5327 \\
\hline & 57 & $90-100$ & 13.2 & 3.6 & $12.2-14.2$ & \\
\hline \multicolumn{7}{|l|}{ Clinical aspects } \\
\hline \multirow[t]{2}{*}{ Mutation type } & 25 & TSC1 & 12.4 & 4.5 & $10.6-14.3$ & 0.791 \\
\hline & 31 & TSC2 & 12.3 & 3.6 & 10.9-13.6 & \\
\hline \multirow[t]{2}{*}{ Active epilepsy } & 50 & Yes & 13.2 & 3.8 & $12.2-14.3$ & 0.074 \\
\hline & 71 & No & 11.9 & 4.2 & $10.9-12.9$ & \\
\hline \multirow[t]{2}{*}{ Structural brain } & 86 & Yes & 13.1 & 4.1 & $12.3-14.0$ & $0.004 \dagger$ \\
\hline & 35 & No & 10.7 & 3.5 & $9.5-11.9$ & \\
\hline \multirow[t]{2}{*}{ SEGA } & 51 & Yes & 13.1 & 4.2 & $12.0-14.3$ & 0.046 \\
\hline & 70 & No & 11.9 & 4.0 & $11.0-12.9$ & \\
\hline \multirow[t]{2}{*}{ Neuropsychiatric } & 60 & Yes & 13.7 & 4.0 & $12.6-14.7$ & $0.001+$ \\
\hline & 61 & No & 11.3 & 3.8 & $10.3-12.2$ & \\
\hline \multirow[t]{2}{*}{ AML } & 70 & Yes & 12.3 & 4.3 & $11.3-13.4$ & 0.866 \\
\hline & 51 & No & 12.6 & 3.8 & $11.5-13.7$ & \\
\hline \multirow[t]{2}{*}{ Lymphangioleiomyomatosis } & 12 & Yes & 12.2 & 4.1 & $9.5-14.8$ & 0.878 \\
\hline & 109 & No & 12.5 & 4.1 & $11.7-13.3$ & \\
\hline \multirow[t]{2}{*}{ Skin } & 116 & Yes & 12.3 & 4.0 & $11.6-13.1$ & 0.281 \\
\hline & 5 & No & 14.6 & 5.9 & $7.3-21.9$ & \\
\hline \multirow[t]{3}{*}{ Number of affected organs } & 31 & $1-3$ & 11.4 & 4.1 & $9.8-12.9$ & $0.006 t$ \\
\hline & 73 & $4-6$ & 12.3 & 4.0 & $11.4-13.3$ & \\
\hline & 17 & $7-8$ & 14.8 & 3.6 & $13.0-16.7$ & \\
\hline \multicolumn{7}{|l|}{ Therapeutic aspects } \\
\hline \multirow[t]{2}{*}{ Anti-seizure medication polytherapy ${ }^{a}$} & 53 & Yes & 12.8 & 3.5 & $11.9-13.8$ & 0.487 \\
\hline & 31 & No & 13.6 & 4.1 & $12.0-15.1$ & \\
\hline \multirow[t]{2}{*}{ Everolimus } & 51 & Yes & 12.7 & 4.1 & $11.6-13.9$ & 0.351 \\
\hline & 70 & No & 12.2 & 4.1 & $11.3-13.2$ & \\
\hline \multirow[t]{2}{*}{ LAEP } & 36 & $<35$ & 10.0 & 3.7 & $8.7-11.2$ & $<0.001 \dagger$ \\
\hline & 84 & $\geq 35$ & 13.6 & 3.7 & $12.8-14.4$ & \\
\hline
\end{tabular}

$A M L$ angiomyolipoma, $C I$ confidence interval, $L A E P$ Liverpool Adverse Events Profile, NDDI-E Neurological Disorders Depression Inventory for Epilepsy, ref. reference category, SD standard deviation, SEGA subependymal giant cell astrocytoma, TSC tuberous sclerosis complex, VAS visual analogue scale, y years

ancludes only individuals with TSC-associated epilepsy/seizures

*comparisons corrected for multiple testing using the Benjamin Hochberg false-discovery rate method, $\dagger$ and bold type denotes a $q$-value of $<0.05$

(false-discovery rate)

$\left(f^{2}=0.173\right)$. The number of affected organs, employment, and grade of disability were not significant predictors in the final regression model, which accounted for $65 \%$ of
HRQoL variance $(p<0.001$; Table 5$)$. The minimal clinically relevant difference for QOLIE-31 in TSC is approximately 11.1 points [39]; however, this was 


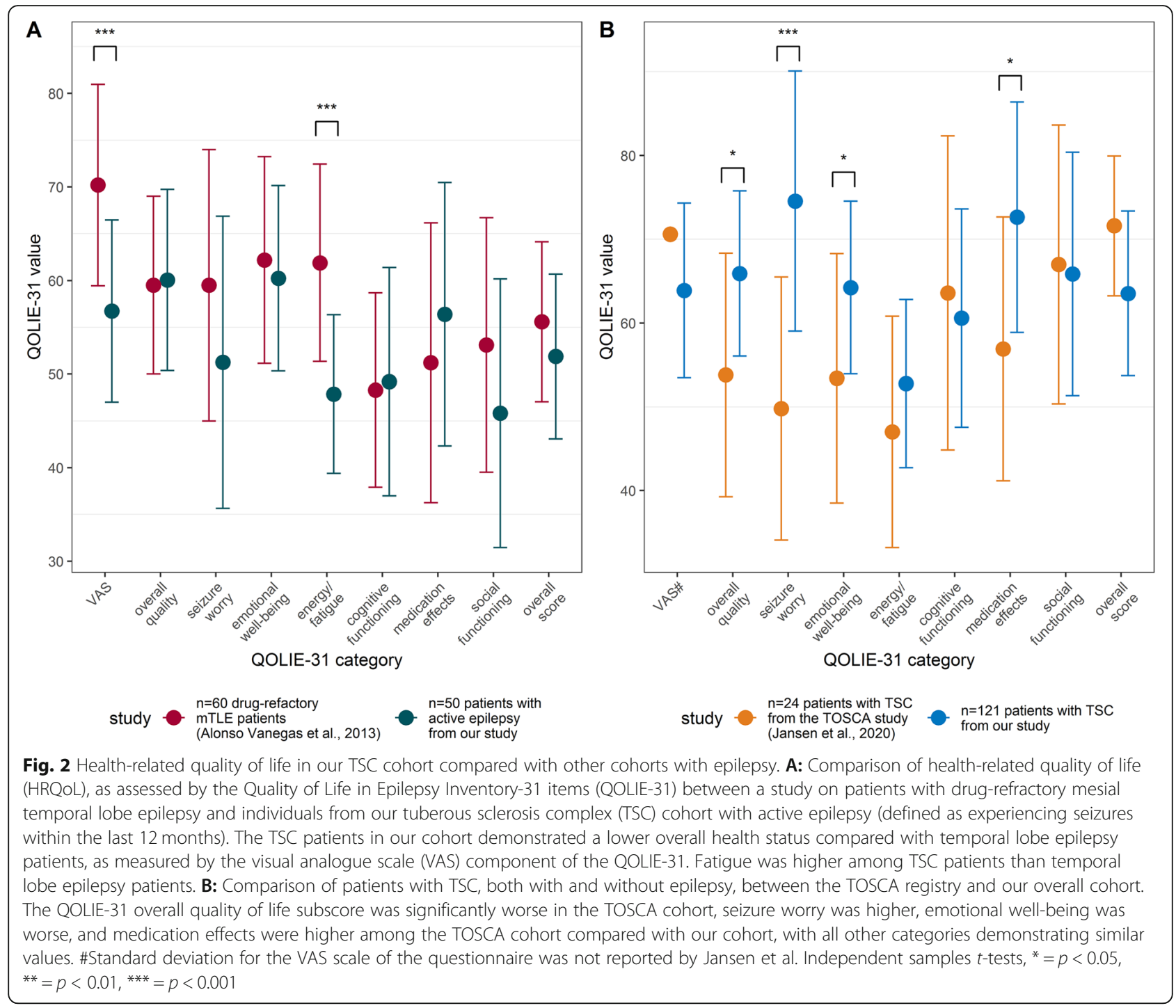

evaluated for longitudinal differences in a solely drugrefractory TSC-epilepsy cohort and is not directly applicable to our group.

\section{Discussion}

This detailed, multicentre QoL study included a large sample of 121 adult individuals with TSC within a single healthcare system and contributed important new information regarding the relationships between clinical and sociodemographic aspects and QoL among individuals with TSC.

Only a few TSC clinical aspects were associated with worse QoL. Specifically, neuropsychiatric manifestations (such as anxiety and depression) and active epilepsy (seizures within the preceding 12 months) reduced QoL. Neuropsychiatric manifestations in TSC are often severe and challenging to treat [5]. It is thus easily comprehensible that QoL among individuals who experience them is worse, however the magnitude of this effect and its persistence in the multivariable analysis was striking. The worsened QoL associated with active epilepsy may be attributable to the persistent burden and social and medical consequences of continuing seizures for the individual, as they are a constant reminder of TSC. By contrast, other manifestations, including renal AML, may only be intermittently burdensome or at later stages, with less effect on QoL.

A higher number of TSC manifestations was associated with reduced QoL, and QoL stratification according to manifestation number provided an insightful look at the range of QoL among individuals with TSC. Those with mild TSC reported QoL comparable to the overall German population, whereas those with multiple manifestations reported substantially worse QoL, similar to severe (more than 8 headache days/month, failure of $\geq 2$ prophylactic medications) chronic migraine or 


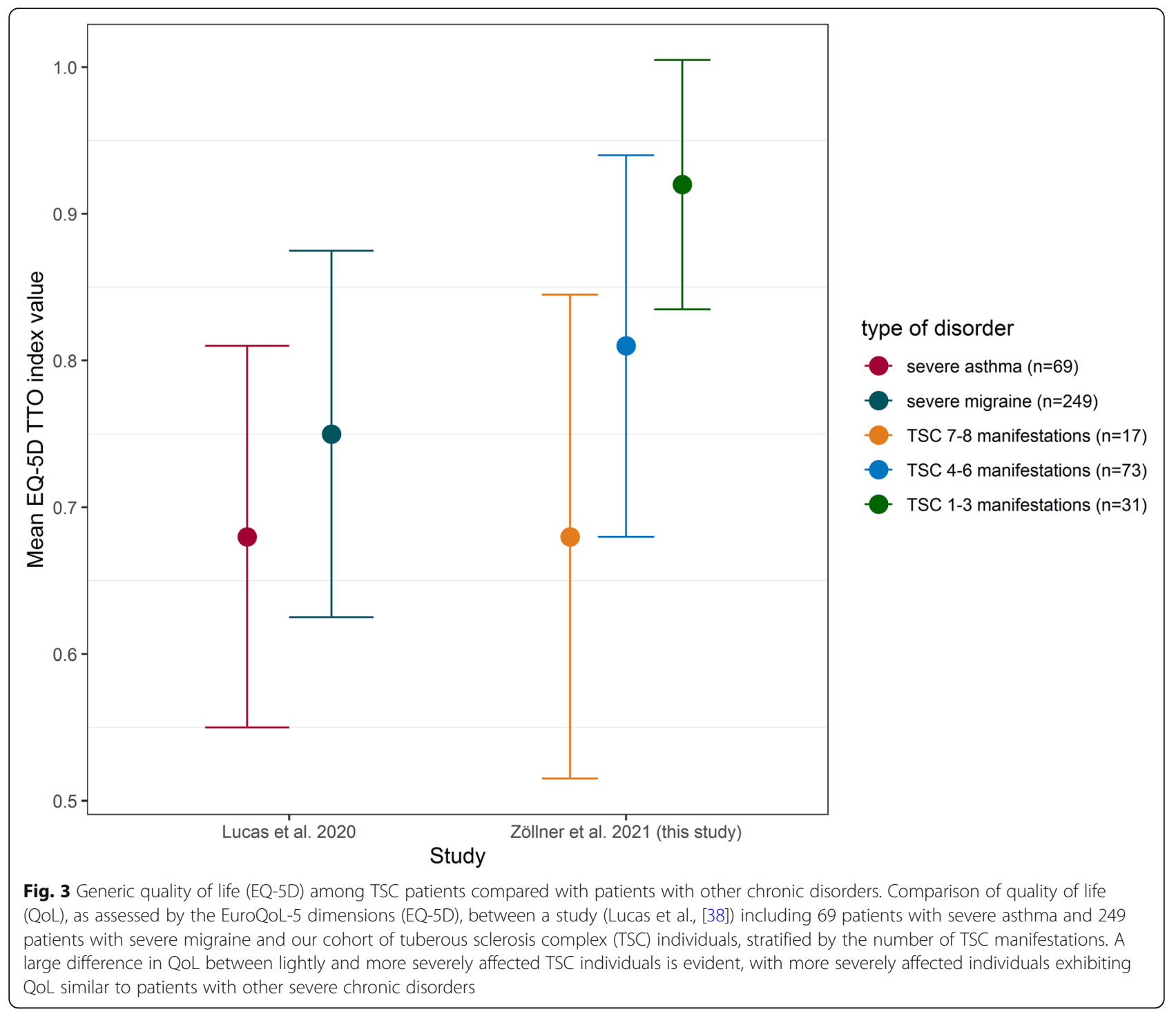

Table 5 Multiple linear regression analyses evaluating potential TSC-related predictors of health-related quality of life (QOLIE-31 overall score)

\begin{tabular}{lllllll}
\hline & Regression coefficient $\boldsymbol{B}$ & $\boldsymbol{S E} \boldsymbol{B}$ & beta & $\boldsymbol{p}$-value & Semi-partial correlation & Tolerance \\
\hline LAEP total score & -0.906 & 0.143 & -0.548 & $<0.001$ & $-0.644 \mid f^{2} 0.709-$ large effect & 0.757 \\
Active epilepsy (yes/no) & -12.608 & 2.810 & -0.358 & $<0.001$ & $-0.511 \mid f^{2} 0.353-$ large effect & 0.885 \\
Neuropsychiatric symptoms (yes/no) & -10.401 & 3.312 & -0.293 & 0.003 & $-0.384 \mid f^{2} 0.173-$ medium effect & 0.644 \\
Number of affected organs & 1.234 & 1.066 & 0.102 & 0.252 & 0.152 & 0.726 \\
Employment (yes/no) & 2.457 & 2.951 & 0.070 & 0.409 & 0.110 & 0.800 \\
Grade of disability ( $\leq 80 />80)$ & -1.081 & 3.390 & -0.030 & 0.751 & -0.042 & 0.615 \\
& Adjusted $R^{2}=0.65 ; F=20.15$, Sig. F. $(p<0.001)$ & & & \\
\hline
\end{tabular}

Variables are ordered according to the standardised $B$ (beta) values, in descending order

LAEP Liverpool Adverse Events Profile, SE standard error. Effect sizes were calculated from the semi-partial correlation as $r_{\text {part }}^{2} /\left(1-r_{\text {part }}^{2}\right.$. Tolerance was used as a measure of predictor multicollinearity, with tolerance values above a threshold of 0.25 indicating no collinearity 
uncontrolled asthma [38]. Therefore, TSC should not be interpreted as a disorder associated with a homogenous QoL simply due to its chronic nature: Distinct differences in QoL were notable, associated with disease burden and neuropsychiatric manifestations.

Therapy-related adverse events reduced QoL among TSC patients, and LAEP was a stronger predictor of reduced QoL in multivariate analysis than neuropsychiatric manifestations or active epilepsy. Although we cannot attribute adverse events to individual medications in this study, neither everolimus nor anti-seizure medication polytherapy [40, 41] was associated with worse QoL when examined individually, suggesting another possible interpretation of the LAEP results. LAEP scores are known to be influenced by the presence of anxiety and depression [42], which are among the most common neuropsychiatric manifestations in adults with TSC $[43,44]$ and were common in our cohort, with $40.5 \%$ affected according to the anxiety domain of the EQ-5D and a mean NDDI-E score of 12.4 (depression highly likely above 13 points). Thus, the LAEP may co-measure affective problems among individuals with TSC.

The perceived TSC-specific burden of illness, as assessed using a 20-item Likert scale, correlated highly with QoL (Fig. 1d), with higher disease burden values significantly associated with reduced QoL. Our scale was constructed as an exploratory tool and has not been previously validated; however, the strong correlation with QoL demonstrates conceptually that a relatively simple questionnaire can capture a broad range of potential burden of illness issues in TSC in a manner that is relevant for assessing QoL.

Although QoL has been used as an outcome variable for some TSC therapy studies (e.g. everolimus [45] and epilepsy surgery [46]), sparse literature addresses QoL in TSC overall. However, a range of studies has recently been published exploring this issue. Amin et al. [14] demonstrated that the psychosocial QoL domain is the most affected in those with TSC, and those with and without epilepsy have worse QoL than the general population. Both findings were supported by our study; the "usual activities" and "anxiety" domains of the EQ-5D were the most commonly affected, and QoL was worse regardless of TSC-associated epilepsy, although those with epilepsy showed comparatively worse QoL. Similar to Vergeer et al., who found worse QoL in those with severe impairment of daily functioning [16], indicators of worse daily functioning, including a higher grade of disability and unemployment, were associated with worse QoL in our study.

Quantitatively, generally higher HRQoL was observed in our cohort than among individuals with TSC from the TOSCA study [12]. However, the QOLIE-31 reporting among this comparator cohort was limited $(n=24)$, making comparisons prone to bias. Among the larger comparator registry patients reporting EQ-5D $(n=143)$, some categories demonstrated more frequent problems than our cohort, including the EQ-5D mobility $(23.8 \%$ vs $12.4 \%$ ) and pain (35\% vs $27 \%$ ) domains, whereas other problems were less frequent than in our cohort. A central issue associated with self-reported QoL assessments is the potential distortion towards a better QoL because more severely affected patients may be unable to complete the questionnaire. Compared with our results, a recent study demonstrated worse overall QoL among individuals with TSC but incorporated a high share of caregiver-reported outcomes [15]. Common QoL questionnaires, including the standard EQ-5D, were not constructed for by-proxy answering, which may introduce the underreporting of issues that rely on internal perception (e.g. pain).

The Epilepsy Stigma Scale was developed to detect stigma among epilepsy patients but features no questions specific to epilepsy. We found that a range of clinical TSC manifestations was associated with severe stigma. However two logically plausible clinical factors (number of TSC manifestations overall and presence of visible skin manifestations) were not associated with severe stigmatisation. On multiple regression, only the LAEP score remained an independent predictor of severe stigma. This may be due to individuals with more severe affective problems (using the LAEP as a proxy instrument) tending to feel more vulnerable and stigmatised. Taken together, these findings imply that affective issues have a stronger influence on feeling stigmatised than actual (potentially visible, and thus identifying) clinical TSC manifestations.

\section{Limitations}

The evaluation of self-reported QoL and burden of illness measures risks under-capturing true QoL due to the exclusion of the most severely affected individuals. However, we found a significant QoL range within our cohort, demonstrating a wide range of participation. In addition, we identified a relevant ceiling effect in the EQ-5D results, indicating that caution remains necessary when using this instrument for clinically heterogeneous disorders such as TSC. An individual psychiatric examination would have provided further insight into the precise contribution of depression or other mental illness to quality of life than was possible in this questionnaire study. The additional survey of the Beck's Depression Inventory would also have allowed a more precise differentiation.

\section{Conclusions}

The severity of TSC-associated seizures, therapy-related adverse events, and neuropsychiatric manifestations, 
particularly anxiety and depression, appear to worsen quality of life among adults with TSC. Further research on quality of life among adult individuals with TSC should focus on differentiating the roles of therapyrelated adverse events from pre-existing affective problems, such as anxiety and depression.

\section{Abbreviations}

AML: Angiomyolipoma; EQ-5D: EuroQoL-5 dimensions; HRQoL: Health-related quality of life; LAEP: Liverpool Adverse Events Profile; NDDI-E: Neurological Disorders Depression Inventory for Epilepsy; QoL: Quality of life; QOLIE31: Quality of Life in Epilepsy Inventory-31 items; SEGA: Subependymal giant cell astrocytoma; TTO: Time-trade-off; TSC: Tuberous sclerosis complex; VAS: Visual analogue scale

\section{Acknowledgements}

The authors would like to thank all of the individuals with TSC who contributed to this survey and the German patient advocacy group Tuberöse Sklerose Deutschland e.V. (https://www.tsdev.org; Wiesbaden, Germany) for their help with participant recruitment.

\section{Authors' contributions}

JPZ, NC, and AS developed the idea for this study. JPZ, JG, and AS collected the data. JPZ, NC, and AS conceived the paper and performed the statistical analysis. JPZ created the charts and figures. All authors wrote the paper, discussed the results, contributed to the final manuscript, and approved the final manuscript for publication.

\section{Funding}

This study was supported by the State of Hessen with a LOEWE-Grant to the CePTER Consortium (https://www.uni-frankfurt.de/67689811).

\section{Availability of data and materials}

The datasets analysed during the current study are available from the corresponding author on reasonable request.

\section{Declarations}

\section{Ethics approval and consent to participate}

This study received ethics approval by the Goethe-University Frankfurt (reference 324/18), and all participants provided informed consent.

\section{Consent for publication}

Not applicable.

\section{Competing interests}

JPZ reports speakers' honoraria and travel grants from Eisai and Desitin Arzneimittel.

FR reports personal fees from Arvelle Therapeutics, Desitin Arzneimittel, Eisai, GW Pharmaceuticals companies, Novartis, Medtronic, Sandoz, Shire, and UCB and grants from the Detlev-Wrobel-Fonds for Epilepsy Research, the Deutsche Forschungsgemeinschaft, the LOEWE Programme of the State of Hesse, and the European Union.

MS reports personal fees from Novartis and GW Pharmaceuticals companies. GK reports personal fees from Desitin Arzneimittel, Eisai, GW Pharmaceuticals companies, UCB, Novartis, Takeda, and Zogenix.

TM reports personal fees and grants from Arvelle Therapeutics, Eisai, GW Pharmaceuticals companies, UCB, and Zogenix.

$\mathrm{CH}$ reports personal fees from Desitin Arzneimittel, Eisai, GW Pharmaceuticals companies, Novartis, Shire, and Zogenix.

AB reports personal fees from Desitin Arzneimittel $\mathrm{GmbH}$, Eisai $\mathrm{GmbH}$, GW Pharma GmbH, Shire/Takeda GmbH, UCB Pharma GmbH, and ViroPharma $\mathrm{GmbH}$.

KMK reports personal fees from UCB Pharma, Novartis Pharma AG, Eisai, and GW Pharmaceuticals and grants from the federal state Hessen, through the LOEWE program, and from the Canadian Institutes of Health Research. SM reports grants from Novartis, UCB, Shire, Deutsche Forschungsgemeinschaft, and Epilepsiestiftung Dr. Wolf. FvP reports personal fees and grants from Bial, Desitin Arzneimittel, Eisai, GW Pharmaceutical companies, Arvelle Therapeutics, Zogenix, and UCB Pharma.
SS-B reports personal fees from Eisai, Desitin Pharma, GW Pharmaceuticals companies, LivaNova, UCB, and Zogenix.

AS reports personal fees and grants from Arvelle Therapeutics, Desitin Arzneimittel, Eisai, GW Pharmaceutical companies, LivaNova, Marinus Pharma, Medtronic, UCB, and Zogenix.

None of the other authors reported any related conflicts of interest.

\section{Author details}

${ }^{1}$ Epilepsy Center Frankfurt Rhine-Main, Center of Neurology and Neurosurgery, Goethe-University Frankfurt, Schleusenweg 2-16 (Haus 95), 60528 Frankfurt am Main, Germany. ${ }^{2}$ Center for Personalized Translational Epilepsy Research (CePTER), Goethe-University Frankfurt, Frankfurt am Main, Germany. ${ }^{3}$ Klinikum Kempten, Klinikverbund Allgäu, Kempten/Allgäu, Germany. ${ }^{4}$ Department of Pediatrics, Klinikum Worms, Worms, Germany. ${ }^{5}$ Department of Pediatrics, University Medicine Mainz, Mainz, Germany. ${ }^{6}$ Epilepsy Center Hessen and Department of Neurology, Philipps-University Marburg, Marburg (Lahn), Germany. ${ }^{7}$ St. Bonifatius Hospital, Lingen, Germany. ${ }^{8}$ Epilepsy Center Kleinwachau, Radeberg, Germany. ${ }^{9}$ Department of Neuropediatrics, Vivantes Klinikum Neukölln, Berlin, Germany. ${ }^{10}$ Department of Neuropediatrics, University Hospital for Children and Adolescents, Rostock, Germany. ${ }^{11}$ Departments of Clinical Neurosciences, Medical Genetics, and Community Health Sciences, Hotchkiss Brain Institute \& Alberta Children's Hospital Research Institute, Cumming School of Medicine, University of Calgary, Calgary, Alberta, Canada. ${ }^{12}$ Department of Pediatric Neurology, Psychosomatics and Pain Management, Klinikum Stuttgart, Stuttgart, Germany. ${ }^{13}$ Department of Neuropediatrics, University Children's Hospital of Saarland, Homburg, Germany. ${ }^{14}$ Department of Neurology, Epilepsy Center, University Medicine Greifswald, Greifswald, Germany. ${ }^{15}$ Division of Nephrology, Medizinische Klinik und Poliklinik IV, Klinikum der LMU München - Innenstadt, Munich, Germany. ${ }^{16}$ Department of Nephrology, Klinikum rechts der Isar, Technische Universität München, Munich, Germany.

${ }^{17}$ Department of Neuropediatrics and Social Pediatrics, Ruhr University Bochum, Bochum, Germany. ${ }^{18}$ Department of Nephrology and Internal Intensive Care, Charité - University Medicine Berlin, Berlin, Germany. ${ }^{19}$ Department of Neuropediatrics, Goethe-University Frankfurt, Frankfurt am Main, Germany. ${ }^{20}$ Institute of Clinical Epidemiology and Biometry, University of Würzburg, Würzburg, Germany.

Received: 26 March 2021 Accepted: 10 May 2021

Published online: 28 June 2021

\section{References}

1. Ebrahimi-Fakhari, D., Mann, L. L., Poryo, M., Graf, N., von Kries, R., Heinrich, B., ... Meyer, S. (2018). Incidence of tuberous sclerosis and age at first diagnosis: new data and emerging trends from a national, prospective surveillance study. Orphanet Journal of Rare Diseases, 13(1), 117. https://doi. org/10.1186/s13023-018-0870-y .

2. Zöllner, J. P., Franz, D. N., Hertzberg, C., Nabbout, R., Rosenow, F., Sauter, M., .. Strzelczyk, A. (2020). A systematic review on the burden of illness in individuals with tuberous sclerosis complex (TSC). Orphanet Journal of Rare Diseases, 15(1), 23. https://doi.org/10.1186/s13023-019-1258-3.

3. O'Callaghan, F. J., Shiell, A. W., Osborne, J. P., \& Martyn, C. N. (1998). Prevalence of tuberous sclerosis estimated by capture-recapture analysis. Lancet, 351(9114), 1490. https://doi.org/10.1016/S0140-6736(05)78872-3

4. Hong, C. H., Tu, H. P., Lin, J. R., \& Lee, C. H. (2016). An estimation of the incidence of tuberous sclerosis complex in a nationwide retrospective cohort study (1997-2010). The British Journal of Dermatology, 174(6), 12821289. https://doi.org/10.1111/bjd.14415

5. Curatolo, P., Moavero, R., \& de Vries, P. J. (2015). Neurological and neuropsychiatric aspects of tuberous sclerosis complex. Lancet Neurology, 14(7), 733-745. https://doi.org/10.1016/S1474-4422(15)00069-1.

6. Józwiak, S., Schwartz, R. A., Janniger, C. K., \& Bielicka-Cymerman, J. (2000). Usefulness of diagnostic criteria of tuberous sclerosis complex in pediatric patients. Journal of Child Neurology, 15(10), 652-659. https://doi.org/10.1177/ 088307380001501003.

7. Chu-Shore, C. J., Major, P., Camposano, S., Muzykewicz, D., \& Thiele, E. A (2010). The natural history of epilepsy in tuberous sclerosis complex. Epilepsia, 51(7), 1236-1241.

8. Kingswood, J. C., Belousova, E., Benedik, M. P., Carter, T., Cottin, V., Curatolo, P., et al. (2020). Renal manifestations of tuberous sclerosis complex: Key findings from the final analysis of the TOSCA study Focussing mainly on 
renal Angiomyolipomas. Frontiers in Neurology, 11, 972. https://doi.org/10.33 89/fneur.2020.00972

9. Kristof, A. S., Zhi Li, P., Major, P., \& Landry, J. S. (2015). Lymphangioleiomyomatosis and tuberous sclerosis complex in Quebec: Prevalence and health-care utilization. Chest, 148(2), 444-449. https://doi. org/10.1378/chest.14-3095

10. Kingswood, J. C., Crawford, P., Johnson, S. R., Sampson, J. R., Shepherd, C., Demuth, D., ... Gray, E. (2016). The economic burden of tuberous sclerosis complex in the UK: A retrospective cohort study in the clinical practice research datalink. Journal of Medical Economics, 19(11), 1087-1098. https:// doi.org/10.1080/13696998.2016.1199432

11. Shepherd, C., Koepp, M., Myland, M., Patel, K., Miglio, C., Siva, V., ... Neary, M. (2017). Understanding the health economic burden of patients with tuberous sclerosis complex (TSC) with epilepsy: A retrospective cohort study in the UK clinical practice research datalink (CPRD). BMJ Open, 7(10), e015236. https://doi.org/10.1136/bmjopen-2016-015236.

12. Jansen, A. C., Vanclooster, S., de Vries, P. J., Fladrowski, C., Beaure d'Augeres, G., Carter, T., et al. (2020). Burden of illness and quality of life in tuberous sclerosis complex: Findings from the TOSCA study. Frontiers in Neurology, 11, 904. https://doi.org/10.3389/fneur.2020.00904

13. Zöllner, J. P., Grau, J., Rosenow, F., Sauter, M., Knuf, M., Kurlemann, G., et al. (2021). Direct and indirect costs and cost-driving factors in adults with tuberous sclerosis complex: A multicenter cohort study and a review of the literature. Orphanet Journal of Rare Diseases, 16(1). https://doi.org/10.1186/ s13023-021-01838-w.

14. Amin, S., Mallick, A. A., Lux, A., \& O'Callaghan, F. (2019). Quality of life in patients with tuberous sclerosis complex (TSC). European Journal of Paediatric Neurology, 23(6), 801-807. https://doi.org/10.1016/j.ejpn.2019.09.006

15. Tritton, T., Bennett, B., Brohan, E., Grant, L., Cooper, A., Fladrowski, C., ... Kingswood, C. (2019). Health utilities and quality of life in individuals with tuberous sclerosis complex (TSC) who experience epileptic seizures: A webbased survey. Epilepsy \& Behavior, 92, 213-220. https://doi.org/10.1016/j. yebeh.2018.11.021

16. Vergeer, M., de Ranitz-Greven, W. L., Neary, M. P., lonescu-Ittu, R., Emond, B., Sheng Duh, M., ... Zonnenberg, B. A. (2019). Epilepsy, impaired functioning, and quality of life in patients with tuberous sclerosis complex. Epilepsia Open, 4(4), 581-592. https://doi.org/10.1002/epi4.12365

17. Northrup, H., Krueger, D. A., \& International tuberous sclerosis complex consensus $G$ (2013). Tuberous sclerosis complex diagnostic criteria update: Recommendations of the 2012 linternational tuberous sclerosis complex consensus conference. Pediatric Neurology, 49(4), 243-254. https://doi.org/1 0.1016/j.pediatrneurol.2013.08.001.

18. Fisher, R. S., Cross, J. H., French, J. A., Higurashi, N., Hirsch, E., Jansen, F. E., et al. (2017). Operational classification of seizure types by the international league against epilepsy: Position paper of the ILAE Commission for Classification and Terminology. Epilepsia, 58(4), 522-530. https://doi.org/1 $0.1111 /$ epi.13670 .

19. Scheffer, I. E., Berkovic, S., Capovilla, G., Connolly, M. B., French, J., Guilhoto, L. , et al. (2017). ILAE classification of the epilepsies: Position paper of the ILAE Commission for Classification and Terminology. Epilepsia, 58(4), 512-521. https://doi.org/10.1111/epi.13709.

20. Willems L.M., Watermann N., Richter S., Kay L., Hermsen A.M., Knake S., ... Strzelczyk A. (2018). Incidence, Risk Factors and Consequences of EpilepsyRelated Injuries and Accidents: A Retrospective, Single Center Study. Frontiers in Neurology, 9, 414. https://doi.org/10.3389/fneur.2018.00414.

21. Riechmann J., Willems L.M., Boor R., Kieslich M., Knake S., Langner C., ... Strzelczyk A. (2019). Quality of life and correlating factors in children, adolescents with epilepsy, and their caregivers: A cross-sectional multicenter study from Germany. Seizure, 69, 92-98. https://doi.org/10.1016/j.seizure.201 9.03.016.

22. Riechmann, J., Strzelczyk, A., Reese, J. P., Boor, R., Stephani, U., Langner, C., .. the EpiPaed Study Group (2015). Costs of epilepsy and cost-driving factors in children, adolescents, and their caregivers in Germany. Epilepsia, 56(9), 1388-1397. https://doi.org/10.1111/epi.13089.

23. Strzelczyk, A., Nickolay, T., Bauer, S., Haag, A., Knake, S., Oertel, W. H., ... Hamer, H. M. (2012). Evaluation of health-care utilization among adult patients with epilepsy in Germany. Epilepsy \& Behavior, 23(4), 451-457. https://doi.org/10.1016/j.yebeh.2012.01.021 .

24. Strzelczyk, A., Schubert-Bast, S., Bast, T., Bettendorf, U., Fiedler, B., Hamer, H. M., ... Rosenow, F. (2019). A multicenter, matched case-control analysis comparing burden-of-illness in Dravet syndrome to refractory epilepsy and seizure remission in patients and caregivers in Germany. Epilepsia, 60(8), 1697-1710. https://doi.org/10.1111/epi.16099

25. Willems, L. M., Richter, S., Watermann, N., Bauer, S., Klein, K. M., Reese, J. P., et al. (2018). Trends in resource utilization and prescription of anticonvulsants for patients with active epilepsy in Germany from 2003 to 2013 - a ten-year overview. Epilepsy \& Behavior, 83, 28-35. https://doi.org/1 0.1016/j.yebeh.2018.03.025

26. May, T. W., Pfäfflin, M., \& Cramer, J. A. (2001). Psychometric properties of the German translation of the QOLIE-31. Epilepsy \& Behavior, 2(2), 106-114. https://doi.org/10.1006/ebeh.2001.0170.

27. Metternich, B., Wagner, K., Buschmann, F., Anger, R., \& Schulze-Bonhage, A. (2012). Depressionsscreening bei Epilepsiepatienten. Zeitschrift für Epileptologie, 25(4), 283-288. https://doi.org/10.1007/s10309-012-0269-z

28. Baker, G. A., Brooks, J., Buck, D., \& Jacoby, A. (2000). The stigma of epilepsy: A European perspective. Epilepsia, 41(1), 98-104. https://doi.org/10.1111/j.1 528-1157.2000.tb01512.x

29. Gilliam, F. G., Barry, J. J., Hermann, B. P., Meador, K. J., Vahle, V., \& Kanner, A. M. (2006). Rapid detection of major depression in epilepsy: A multicentre study. Lancet Neurology, 5(5), 399-405. https://doi.org/10.1016/S1474-4422 (06)70415-X.

30. Romoli, M., Eusebi, P., Siliquini, S., Bedetti, C., Calabresi, P., \& Costa, C. (2018). Liverpool adverse events profile: Italian validation and predictive value for dropout from antiepileptic treatment in people with epilepsy. Epilepsy \& Behavior, 81, 111-114. https://doi.org/10.1016/j.yebeh.2018.01.028

31. Ellis, P. D. (2010). The essential guide to effect sizes: Statistical power, metaanalysis, and the interpretation of research results. Cambridge: Cambridge University Press. https://doi.org/10.1017/CBO9780511761676

32. Miles, J., \& Shevlin, M. (2001). Applying regression and correlation: a guide for students and researchers. London: SAGE Publications.

33. Benjamini, Y., \& Hochberg, Y. (1995). Controlling the false discovery rate: A practical and powerful approach to multiple testing. Journal of the Royal Statistical Society: Series B (Methodological), 57(1), 289-300.

34. Alonso-Vanegas, M. A., Cisneros-Franco, J. M., Castillo-Montoya, C., MartinezRosas, A. R., Gomez-Perez, M. E., \& Rubio-Donnadieu, F. (2013). Self-reported quality of life in pharmacoresistant temporal lobe epilepsy: Correlation with clinical variables and memory evaluation. Epileptic Disorders, 15(3), 263-271. https://doi.org/10.1684/epd.2013.0590 .

35. Quality of Life in Epilepsy Scoring Manual [https://www.rand.org/content/da m/rand/www/external/health/surveys_tools/qolie/qolie31_scoring.pdf]. Accessed 1 Mar 2021.

36. Devinsky, O., Vickrey, B. G., Cramer, J., Perrine, K., Hermann, B., Meador, K., \& Hays, R. D. (1995). Development of the quality of life in epilepsy inventory. Epilepsia, 36(11), 1089-1104. https://doi.org/10.1111/j.1528-1157.1995.tb004 67.x.

37. Huber, M. B., Felix, J., Vogelmann, M., \& Leidl, R. (2017). Health-Related Quality of Life of the General German Population in 2015: Results from the EQ-5D-5L. International Journal of Environmental Research and Public Health, 14(4), 426. https://doi.org/10.3390/ijerph14040426.

38. Lucas, C., Aly, S., Touboul, C., Sellami, R., Guillaume, X., \& Garcia, G. (2020). Patient-reported outcome in two chronic diseases: A comparison of quality of life and response profiles in severe migraine and severe asthma. Patient Related Outcome Measures, 11, 27-37. https://doi.org/10.2147/PROM.S222597.

39. de Vries, P. J., Franz, D. N., Curatolo, P., Nabbout, R., Neary, M., Herbst, F., .. Lawson, J. A. (2018). Measuring health-related quality of life in tuberous sclerosis complex - psychometric evaluation of three instruments in individuals with refractory epilepsy. Frontiers in Pharmacology, 9, 964. https:// doi.org/10.3389/fphar.2018.00964

40. Strzelczyk, A., Grau, J., Bast, T., Bertsche, A., Bettendorf, U., Hahn, A., .. Zöllner, J. P. (2021). Prescription patterns of antiseizure drugs in tuberous sclerosis complex (TSC)-associated epilepsy: A multicenter cohort study from Germany and review of the literature. Expert Review of Clinical Pharmacology. https://doi.org/10.1080/17512433.2021.1911643.

41. Stockinger J., Strzelczyk A., Nemecek A., Cicanic M., Bösebeck F., Brandt C., ... Steinhoff B.J. (2021). Everolimus in adult tuberous sclerosis complex patients with epilepsy: Too late for success? A retrospective study. Epilepsia, 62, 785794. https://doi.org/10.1111/epi.16829.

42. Panelli, R. J., Kilpatrick, C., Moore, S. M., Matkovic, Z., D'Souza, W. J., \& O'Brien, T. J. (2007). The Liverpool adverse events profile: Relation to AED use and mood. Epilepsia, 48(3), 456-463. https://doi.org/10.1111/j.1528-1167.2006.00956.x .

43. Mowrey, K. E., Ashfaq, M., Pearson, D. A., Hashmi, S. S., Roberds, S. L., Farach, L. S., \& Northrup, H. (2019). The impact of psychiatric symptoms on tuberous 
sclerosis complex and utilization of mental health treatment. Pediatric Neurology, 91, 41-49. https://doi.org/10.1016/.jpediatrneurol.2018.10.011.

44. Pulsifer, M. B., Winterkorn, E. B., \& Thiele, E. A. (2007). Psychological profile of adults with tuberous sclerosis complex. Epilepsy \& Behavior, 10(3), 402-406. https://doi.org/10.1016/j.yebeh.2007.02.004 .

45. Krueger, D. A., Wilfong, A. A., Holland-Bouley, K., Anderson, A. E., Agricola, K., Tudor, C., ... Franz, D. N. (2013). Everolimus treatment of refractory epilepsy in tuberous sclerosis complex. Annals of Neurology, 74(5), 679-687. https:// doi.org/10.1002/ana.23960

46. Liu, S., Yu, T., Guan, Y., Zhang, K., Ding, P., Chen, L., ... Liang, S. (2020). Resective epilepsy surgery in tuberous sclerosis complex: A nationwide multicentre retrospective study from China. Brain, 143(2), 570-581. https:// doi.org/10.1093/brain/awz411

\section{Publisher's Note}

Springer Nature remains neutral with regard to jurisdictional claims in published maps and institutional affiliations.

Ready to submit your research? Choose BMC and benefit from:

- fast, convenient online submission

- thorough peer review by experienced researchers in your field

- rapid publication on acceptance

- support for research data, including large and complex data types

- gold Open Access which fosters wider collaboration and increased citations

- maximum visibility for your research: over $100 \mathrm{M}$ website views per year

At BMC, research is always in progress.

Learn more biomedcentral.com/submissions 